9

\title{
TECNOLOGIA E DESIGN NA JUSTIÇA BRASILEIRA: \\ O PIONEIRISMO DO IJUSPLAB
}

\section{TECHNOLOGY AND DESIGN IN BRAZILIAN JUSTICE: IJUSPLAB PIONEERING}

Alexandre Zavaglia Coelho*

\section{RESUMO}

A quantidade de dados e o uso de novas tecnologias está transformando o universo jurídico. Além das oportunidades de novos serviços jurídicos trazidas pelo impacto do uso da tecnologia no dia a dia da sociedade e, assim, no direito material, temos à disposição novas ferramentas tecnológicas para aprimorar as atividades cotidianas dos escritórios de advocacia, departamentos jurídicos, do Poder Judiciário e demais instituições públicas e privadas, que estão transformando o modelo de organização dos agentes que integram o sistema de justiça. Para uma discussão mais aprofundada sobre os desafios e as oportunidades que a inovação e o uso de tecnologia podem nos trazer, é preciso conscientizar os juristas sobre certa confusão entre conceitos de (a) direito digital, (b) automação e (c) ciência de dados, bem como sobre o uso de (d) técnicas de design na área do direito. $\mathrm{E}$ entre as iniciativas de maior sucesso do poder público que integram esses conceitos para a construção e o desenvolvimento

* Mestre em direito pela Puc-Campinas e doutorando pela Unicamp, CEO da Future Law. Advogado especializado em projetos de ciência de dados e uso de técnicas de design para o suporte a decisão na solução de problemas jurídicos, Coordenador do Curso de Ciência de dados aplicada ao Direito e um dos coordenadores da RDTec. Revista Direito e Novas Tecnologias, RT. 
de projetos para a modernização dos serviços públicos está o iJuspLab da Justiça Federal de São Paulo. Ao usar as técnicas mais avançadas de Legal Desgin, o laboratório integra esses conceitos e promove a participação efetiva de magistrados, servidores, cidadãos e demais stakeholders na colaboração entre essas diferentes visões dos mesmos problemas, na busca pelo amplo acesso à justiça.

Palavras-chave: Direito digital; Ciência de dados; Design; Legal design; iJuspLab; Inteligência artificial; Dados; Big data; Direito e tecnologia.

\begin{abstract}
The amount of data and the use of new technologies is transforming the legal universe. In addition to the opportunities for new legal services brought about by the impact of the use of technology on society's daily life and, thus, in material law, we have at our disposal new technological tools to improve the daily activities of law firms, legal departments, the judiciary and other public and private institutions, which are transforming the organization model of the agents that make up the justice system. For a more in-depth discussion of the challenges and opportunities that innovation and the use of technology can bring us, it is necessary to raise awareness about some confusion between (a) digital law, (b) automation, and (c) computer science. as well as the use of (d) design techniques in the area of law. And among the most successful government initiatives that integrate these concepts for the construction and development of projects for the modernization of public services is the iJuspLab of the Federal Justice of São Paulo. Using the most advanced Legal Desgin techniques, the laboratory integrates these concepts and promotes the effective participation of magistrates, servants, citizens and other stakeholders in collaboration between these different views of the same issues, in the pursuit of broad access to justice.
\end{abstract}

Keywords: Digital law; Data science; Design; Legal design; iJuspLab; Artificial intelligence; Data; Big data; Law and technology. 


\section{DIREITO DIGITAL, AUTOMAÇÃO, CIÊNCIA E DADOS E DESIGN NA ÁREA DO DIREITO}

As novas dinâmicas sociais e o uso crescente de tecnologia nas atividades cotidianas têm demandado grandes transformações na forma como organizamos as instituiçõos do universo jurídico.

De um lado, temos o crescente impacto da tecnologia na vida da sociedade e nos conflitos inerentes a esse fenômeno - e no direito material, o que demanda a qualificação dos profissionais para essas novas temáticas.

Por outro, a necessidade de aprimorar os serviços jurídicos pelo uso de ferramentas tecnológicas de última geração, para modernizar os procedimentos internos e otimizar os resultados do trabalho em escritórios de advocacia, departamentos jurídicos, no Poder Judiciário, no Ministério Público, e assim por todas as carreiras jurídicas.

Para uma discussão mais aprofundada sobre os desafios e as oportunidades que a inovação e o uso de tecnologia podem nos trazer, é preciso conscientizar os juristas sobre certa confusão entre conceitos de (a) direito digital, (b) automação e (c) ciência de dados, bem como sobre o uso de (d) técnicas de design na área do direito.

$\mathrm{O}$ (a) direito digital, apesar da importância da expressão, não é nem será uma área autônoma do Direito. $\mathrm{O}$ uso de tecnologia está crescendo exponencialmente em todas as áreas dos setores público e privado, para os mais variados assuntos e tarefas. Dessa maneira, ao mesmo tempo que são muitos os benefícios pelo uso das inovações, também são gerados novos conflitos quase que diariamente e, assim, diversos reflexos no direito material e na jurisprudência.

E nunca fará sentido colocar todos esses novos temas em um "código de direito digital", especialmente pelo fato de que cada ramo tem seus princípios próprios, e o in dubio pro reo e a verdade real do Penal são bem diferentes da aceitação da verdade formal pelo Processo Civil, por exemplo.

Então, fora os assuntos gerais tratados pelo Marco Civil da Internet e pela legislação extravagante, as discussões sobre a responsabilidade do carro autônomo continuarão no Direito Civil; o crime de invadir dispositivo informático (art. 154A CP) continuará no Direito Penal; o uso de patinetes elétrico nas ruas no Direito Administrativo e dentro dos ambientes de regulação da mobilidade urbana; a proteção de dados pessoais será tratada pelos princípios de Direito Constitucional, nas leis específicas sobre a questão (LGDP) e demais áreas reflexas (direito do consumidor, por exemplo), e assim por diante. 
•- Inovação no Judiciário

E não faltará trabalho para quem se especializar nos reflexos da tecnologia dentro de suas próprias áreas de atuação, pois a demanda por profissionais do direito para auxiliar nessas questões só tem aumentado e são muitas as vagas e as oportuidades para especialistas qualificados.

A (b) automação, por sua vez, é o uso de tecnologia para facilitar as atividades humanas, para organizar os seus procedimentos por meio do uso de dispositivos eletrônicos ou mecânicos.

No nosso caso, o melhor exemplo é o processo eletrônico. Os atos e as atividades dos atores do processo continuam os mesmos, mas agora todo esse workflow está automatizado, e o impulso oficial é respeitado pelo apoio da tecnologia não só no cumprimento das obrigações de cada parte nesse todo, mas na integração entre os atos processuais, seus responsáveis e as fases do processo.

Assim, temos praticamente todos os novos processos em ambiente digitalizado, e a possibilidade de gestão do conhecimento de cada demanda judicial, o potencial de entender o tempo de duração dos atos, os temas mais discutidos, a doutrina e jurispruência citados, e quais são as decisões de forma muito individualizada. Mas, apesar de todas essas alternativas, podemos simplesmente não fazer nada com esses dados.

A automação otimiza as tarefas e a ciência de dados nos auxilia a organizar esses dados gerados no ambiente digitalizado para apoiar a tomada de decisão. $\mathrm{Ou}$ seja, enquanto a automação gera o ambiente digitalizado que permite o uso desses dados, a aplicação de técnicas de ciência de dados permite transformar milhares de dados (big data) em informação, de modo que os seus conceitos e finalidades são outros, mas interligados e complementares. Até os profissionais de tecnologia que atuam na automação e com ciência de dados costumam ser diferentes.

A (c) ciência de dados não é algo novo. Consiste basicamente na organização de dados de fontes variadas, sua compilação, padronização e cruzamento para que os resultados ou seus insights possam auxiliar na tomada de decisão, na resolução de problemas em todas as áreas do saber, para trazer evidências científicas e informaçôes objetivas para balizar decisões humanas.

Desde seus primeiros tratados em 1.950, a ciência de dados avançou muito nos últimos anos e se tornou uma das mais relevantes áreas da ciência moderna. A diminuição de custo de armazenamento de dados, a capacidade de processamento e cruzamento com o uso de novas tecnologias, e o ambiente digitalizado criado pela internet em conjunto com a produção de conteúdo - principalmente por meio de dispositivos móveis, criou um ambiente muito propício para essa evolução. 
Após o uso dessas técnicas no setor de marketing digital, depois streamings de músicas, filmes, e assim expandindo sua aplicação para todos os setores da economia, a área do direito tem se beneficiado de forma crescente pela utilização dessas técnicas.

$\mathrm{Na}$ verdade, o uso de ciência de dados está auxiliando a criar uma espécie de radiologia (raio X) da aplicação do direito. Com milhares de processos físicos, não tínhamos a oportunidade de compreender exatamente e em detalhes os problemas refletidos nos processos, até porque os dados cadastrados para cada demanda são geralmente despadronizados e incompletos, e os ambientes de pesquisa, apesar de digitalizados, não nos permitem uma visão mais exata e aprofundada desse todo.

Por meio dessas técnicas, por exemplo, agora é possível organizar a base de dados de um determinado tema e compreender em detalhes, com evidências científicas, como estão sendo decididos esses casos relacionados e qual a tendência do judiciário sobre o assunto, a média de acordos realizados por localidade, o tempo de duração, entre muitos outros indicadores que aumentam muito a assertividade e os resultados dos serviços jurídicos relacionados.

E tudo isso tem sido usado não apenas para entender as demandas judiciais e extrajudiciais, mas também para uma mudança na cultura da gestão e na forma de solucionar problemas jurídicos.

É como se tivessemos agora à disposição um exame detalhado, em um cenário em que a tecnologia (softwares ou "robos") é a máquina de raio-x; o cientista de dados (que não é mais uma pessoa, mas um time multidisciplinar que necessita da participação de profissionais experientes da área do direito) funciona como o radiologista, que organiza as imagens e informaçōes e confecciona um laudo; para que o médico (o advogado, juiz, promotor etc.) defina o tratamento ou as atividades necessárias até a solução do problema.

Nesse cenário, fica claro que a tecnologia e a ciência de dados são apenas um ferramental para otimizar as tarefas e auxiliar a tomada de decisão, para que o jurista continue a exercer o seu papel imprescindível no sistema de justiça, seja qual for a sua carreira. Por isso que a tecnologia não coloca em risco ou pretende acabar com o Direito, pelo contrário, pois se usada corretamente servirá apenas para potencializar tudo o que fazemos.

$\mathrm{Na}$ verdade, estamos em um momento em que é preciso ainda organizar as informações, as bases de dados, para entender quais são os indicadores capazes de balizar a tomada de decisão, para a definição da trajetória e das ferramentas necessárias para solucionar os mais complexos problemas jurídicos. 
•- Inovação no Judiciário

E em ambientes de rápidas mudanças, como esse que estamos vivenciando, são naturais as preocupações sobre como agir, como se preparar, sobre como implementar essas melhorias e inserir soluçōes tecnológicas em nossas atividades profissionais.

Até porque, tudo isso está acontecendo agora e tem que ser feito em harmonia com uma regra básica: a tecnologia só funciona para resolver nossas necessidades e a partir da definição das diretrizes dadas pelos seres humanos.

Ao passo que a automação e a digitalização avançam, e muitas tarefas passam a ser realizadas com o auxílio dessas inovações, existem muitas oportunidades nesse movimento, tanto para as organizações como para um rápido crescimento profissional.

Por isso é que o grande desafio do mundo atual é a formação e a adaptação dos profissionais a essa nova realidade. Se tem algo que aprendemos nesses últimos anos, é que nada funciona sem as pessoas.

Nada funciona sem os profissionais experientes de cada assunto para criar e desenvolver essas inovações, sem aqueles que serão seus usuários e, principalmente, os que vão analisar todas as informações e transformá-las em resultado.

E é aí que entra o (d) design. O conceito de design vai muito além da parte estética de um objeto, de um software ou de metodologias para sua aplicação como design thinking, agile, entre outras.

Está ligado às estrategicas e funcionalidades para solucionar um problema, desde o entendimento e a observação, a definição sobre quais as ferramentas e os melhores caminhos dessa trilha, até o protótipo e os testes necessários para garantir a sua eficácia. E, mesmo que de forma inata ou insconsciente, até pelo dom natural do profissional do direito, sempre nos utilizamos desses conceitos nos serviços jurídicos.

Mas foi nos últimos anos que começamos a compreender melhor essas técnicas e como nos valer de seus princípsio, por meio de uma nova área que está sendo difundida internacionalmente no mundo jurídico como Legal Design.

Para Margareth Hagan, uma das maiores expoentes na pesquisa e aplicação de técnicas de design na área do direito ${ }^{1}$ :

"Legal design is the application of human-centered design to the world of law, to make legal systems and services more human-centered, usable, and satisfying. (...) Design offers methods and priorities to transform the legal sector, to make legal outcomes more aligned with those its users desire, and to create ambitious new visions

\footnotetext{
HAGAN, Margareth. Law By Design. Disponível em: <http://www.lawbydesign.co/en/legal-
} -design/, Acesso em: maio de 2019. 
for how legal services can be provided. A design approach to legal services puts people and their contexts as the focus, questions how their status quo could be improved, and then considers the potential of technology as an intervention."

Se o direito material está se adaptando a essas novas demandas oriundas do uso de automação, de inteligência artificial e da análise de big data no dia a dia da sociedade; se a automação e as técnicas de ciências de dados estão nos auxiliando a otimizar a produção de bens e serviços e a resolver os nossos problemas cotidianos; é certo que o uso das técnicas mais avançadas de design também estão, por sua vez, ajudando a colocar o ser humano como o centro de todo esse movimento.

Até porque inovação não é necessariamente algo somente ligado a tecnologia informática e, nosso caso, também tem muito a ver com a tecnologia jurídica e a novas formas de pensar e agir no mundo do Direito.

E as possibilidades do Legal Design são infinitas, e estão sendo pesquisadas por essa nova disciplina que integra as seguintes sub-áreas:

Figura 1. Áreas do legal design.

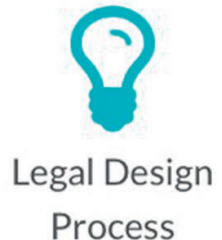

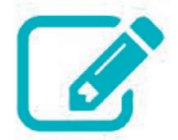

Visual Law

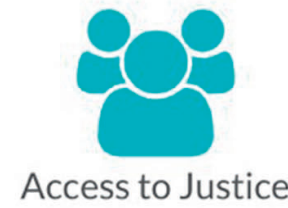

Access to Justice

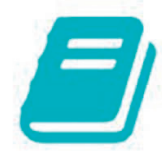

Legal Ed \&

Practice

Fonte: Disponível em: http://www.legaltechdesign.com/.

Assim, o Legal Design estuda (i) os processos organizacionais, tanto no setor público como privado, e como (re) desenhar os serviços jurídicos; como (ii) entregar as informações jurídicas de acordo com o entendimento e as necessidades do destinatário de cada serviço ou atividade; (iii) o acesso à justiça em seus sentido mais amplo, não só como acesso ao sistema judicial, mas principalmente sobre como garantir a tutela e o acesso aos direitos envolvidos; assim como (iv) a formação dos novos juristas e a prática e educação permanente dos profissionais, que estão no mercado para essas novas habilidades e competências.

Nesse ponto, vale lembrar os ensinamentos da clássica obra, e ao mesmo tempo sempre atual, Acesso à Justiça, de CAPPELLETTI e GARTH ${ }^{2}$, por meio da

2 CAPPELLETTI, Mauro e GARTH, Bryant. Acesso à justiça. Porto Alegre: Fabris, 1988, p. 12. 


\section{•- Inovação no Judiciário}

qual aprendemos que o acesso à justiça deve "ser encarado como o requisito fundamental - o mais básico dos direitos humanos - de um sistema jurídico moderno e igualitário que pretende garantir, e não apenas proclamar os direitos de todos".

Pois se formos analisar profundamente esse fenômeno, vamos compreender que todo esse arcabouço de adaptação do direito à essa nova realidade, de uso de tecnologia de última geração para automação de tarefas e análise de dados, e a aplicação de técnicas de design para a solução de problemas jurídicos, se integram pela busca pelo acesso à justiça, do melhor caminho para dar a cada um o que lhe é de direito.

E assim temos visto muitas iniciativas no setor privado, nos escritórios de advocacia e nos departamentos jurídicos das empresas, pelo uso de ferramentas de gestão, de automação de documentos, de plataformas de acordo, de analytics (análise e cruzamentos de dados), na criação de políticas de acordo e de provisão bastante individualizadas para ampliar a base de resolução de conflitos por meios alternativos, assim como para melhorar a definição sobre qual a melhor tese ou o entendimento da jurisprudência sobre cada tema.

Ou seja, iniciativas que buscam transformar o jurídico de custos e problemas, em um ambiente de ativos estratégicos para a gestão, para sair de um modelo reativo, que espera as demandas judiciais para então buscar a solução, para um cenário proativo, que antecipa os caminhos (a partir do entendimento dos problemas pelos dados - analytics) e amplia as atividades de melhoria de procedimentos internos para evitar processos por motivos recorrentes, de mediação, negociação e o aprimoramento de políticas de acordo e uso de plataformas digitais para essa finalidade.

Também para dar subsídios às atividades relacionadas aos processos judiciais, não como um fim, mas como um meio para orientar as estratégias jurídicas.

E o poder público, apesar das dificuldades inerentes ao orçamento e formas de contratação dessas inovações tecnológicas, também tem demonstrado grande avanço nesse contexto, com muitas iniciativas em desenvolvimento nos Tribunais Superiores (STF, STJ, TSE e no TST) e demais setores do Poder Judiciário, nos órgãos de controle como CGU e TCU, na PGFN, no Ministério Público, na Polícia Federal, entre diversos outros.

E entre as iniciativas de maior sucesso do poder público, e que tem se transformado em um verdadeiro modelo para o Poder Judiciário, está o iJuspLab da Justiça Federal de São Paulo. 


\section{O iJuspLab}

Fundado em 12 de junho de 2.017, por meio de uma iniciativa da Diretoria Geral do Foro da Seção Judiciária de São Paulo da Justiça Federal, o iJuspLab é o primeiro laboratório de inovação do Poder Judiciário brasileiro.

A ideia surgiu em 2016, "período em que a Justiça Federal de São Paulo enfrentava diversos desafios com a drástica redução orçamentária, problemas com centralização administrativa, dificuldades de comunicação (interna e externa), improvisação e carência de dados administrativos"'.

Com sede no Fórum Federal Ministro Pedro Lessa da cidade de São Paulo, localizado na Avenida Paulista, o espaço moderno e com o ambiente adequado para o desenvolvimento de atividades de colaboração e uso de técnicas de design para a área do Direito, foi criado para ultrapassar os métodos tradicionais de gestão dos problemas sociais e das demandas do Poder Judiciário, para incentivar a criatividade e a cooperação.

Segundo o juiz federal Paulo Cezar Neves Junior, um dos fundadores e diretor do foro no momento de sua inauguraçãa ${ }^{4}$ :

"Esses tipos de laboratórios são uma nova forma institucional de enfrentarmos os desafios do setor público de maneira bem diferente dos métodos tradicionais. Trata-se de um espaço colaborativo e criativo para troca de conhecimento e ideias, com o objetivo de oferecer condiçôes para que a inovação ocorra de forma contínua numa determinada organização"

Segundo as diretrizes do projeto, ao utilizar métodos multidisciplinares, as atividades do laboratório integram magistrados, servidores, cidadãos e demais stakeholders na colaboração entre essas diferentes visões dos mesmos problemas, para a eliminação da hierarquia na construção coletiva de novas formas e modelos para a prestação de serviços pelo Poder Judiciário.

Ao usar as técnicas mais modernas de Legal Desgin, o laboratório integra todas as questóes discutidas acima e promove a participação efetiva desses atores, na busca pela modernização dos serviços e do acesso amplo à justiça.

\footnotetext{
3 Disponível em: <http://web.trf3.jus.br/noticias/Noticias/Noticia/Exibir/355320>. Acesso em: maio de 2019.

4 Idem.
} 
•• Inovação no Judiciário

Para a juíza federal Luciana Ortiz Zanoni, então vice-diretora no momento de sua fundação e atual diretora do foro, o laboratório é:

"Um espaço de participação democrática. Nele, juízes, servidores, operadores do direito e usuários da Justiça, poderão desenvolver, com o uso de técnicas e metodologias próprias, projetos e ações que visem um serviço público de maior qualidade”.

Entre as várias atividades, estão o apoio ao projeto do CNJ - Conselho Nacional de Justiça para mapear açôes desenvolvidas pelo Judiciário no alcance aos objetivos de desenvolvimento sustentável - ODS da ONU ${ }^{6}$, o MOI - mapeamento de oportunidades de inovação e a criação de um Radar de Inovação para entender o "atual estado de prontidão para inovar da JFSP com relação aos temas Governança, Métricas, Cultura, Aprendizado e Gestão da Informação” e, assim, acompanhar o avanço dessa percepção sobre a inserção dessas iniciativas na Justiça Federal e demonstrar a importância do avanço de projetos como a Rede de colaboração:

Figura 1. Radar de inovação - cruzamento geral.

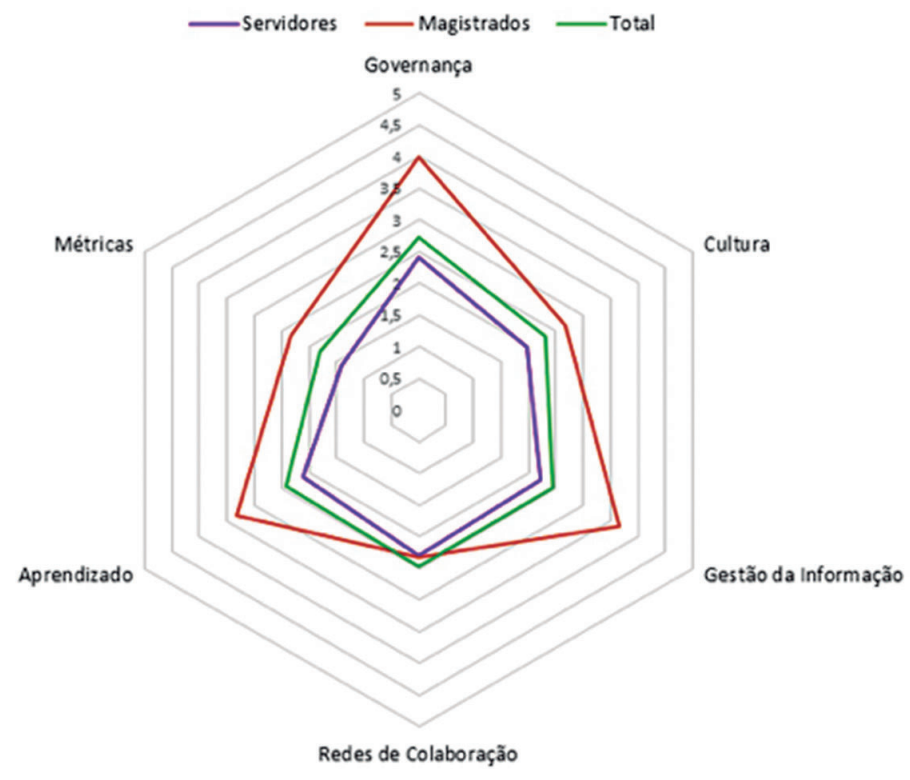

Fonte: Justiça Federal de Primeiro Grau em São Paulo.

5 Disponível em: <http://web.trf3.jus.br/noticias/Noticias/Noticia/Exibir/355320>. Acesso em: maio de 2019.

6 Disponível em: $<$ https://www.youtube.com/watch?v=Dsdp8ZS3ojk\&feature=youtu.be $>$. Acesso em: maio de 2019.

7 Disponível em: <http://www.jfsp.jus.br/moi/>. Acesso em: maio de 2019. 
Também tem sido feito um trabalho muito relevante de levantamento de dados de gestão, tanto para reorganização do orçamento como para entender e definir indicadores e parâmetros para contratações futuras, ou mesmo para compreender a demanda projetada para o próprio judiciário nos próximos anos, para melhor organização dos recursos humanos e financeiros nas diferentes comarcas.

E os resultados do laboratório têm sido muito relevantes, a ponto de se tornar referência nacional de inovação dentro do Poder Judiciário, com muitas oportunidades para a organização interna e para o relacionamento com os demais envolvidos.

Nesse contexto, verifica-se que existem muitas áreas em que é possível "atuar em conjunto com atores internos e externos ao Poder Judiciário", e que "existem várias oportunidades junto ao $\mathrm{CNJ}$, à advocacia, ao Ministério Público, inclusive envolvendo também empresas e os jurisdicionados em geral", como afirmou o atual vice-diretor do Foro da Seção Judiciária de São Paulo, Caio Moysés de Lima. ${ }^{8}$

E essa cocriação horizontal de novos serviços incentiva as ideias e a troca de experiências. Por meio da utilização da metodologia de Design thinking, esse ambiente proporciona "uma abordagem prática e centrada na experiência do usuário, que permite responder criativamente às necessidades, através do trabalho colaborativo entre equipes multidisciplinares" e em etapas de imersão, ideação e prototipagem, para a geração e o teste dessas soluções inovadoras?.

Nesse caminho, foram muitas atividades e serviços como o Inovaday, Meetups, oficinas, o Banco de Soluções IJuspLab, a Caixa de ferramentas (toolkit), boletins de percepção de inovações, geração de conteúdo especializado, a Rede de Inovação no Setor Público, o Observatório de Inovação do Setor Público da OCDE (OPSI) e o projeto "apresente seu problema"10.

E ao integrar a formação e a capacitação dos magistrados e servidores, a inserção de conceitos e projetos de automação e ciência de dados, e a utilização de técnicas de design com a troca de ideias e experiências com outros órgãos e os cidadãos para aprimorar os serviços do Poder Judiciário, o iJuspLab é o exemplo real de que vale a pena investir em inovação.

8 Disponível em: <http://www.jfsp.jus.br/comunicacao-publica/indice-noticias/noticias-2018/ 10122018-ijusplab-recebe-visita-de-conselheira-do-cnj-e-estudantes/>. Acesso em: maio de 2019.

9 Disponível em: <http://www.jfsp.jus.br/inovajusp/ijusplab/o-que-fazemos/>. Acesso em: maio de 2019.

10 Disponível em: <http://www.jfsp.jus.br/carta/>. Acesso em: maio de 2019. 
•• Inovação no Judiciário

E essa iniciativa também demonstra, na prática, que nada acontece sem as pessoas, que realmente estão no centro dessa revolução que estamos vivenciando, e são a verdadeira razão para a cocriação de todas essas novas soluções.

Alcançamos o momento de ultrapassar as manchetes sensacionalistas e os conceitos equivocados sobre as relações entre o Direito e as inovações, sejam elas tecnológicas ou não. E avançar em agendas concretas e objetivas, com discussões e projetos práticos que vão, respeitadas as questões culturais e o tempo necessário para essa evolução, demonstrar que todas essas novas ferramentas tecnológicas e o uso de técnicas de design estão à serviço do Direito, das instituições e dos profissionais da área, como um ferramental para aprimorar os serviços jurídicos e alcançar o tão almejado acesso à justiça.

E o iJuspLab é a expressão de tudo o que existe de melhor nesse movimento.

\section{BIBLIOGRAFIA}

CAPPELlETTI, Mauro e GARTH, Bryant. Acesso à Justiça. Porto Alegre: Fabris, 1988, p. 12.

HAGAN, Margareth. Law by design. Disponível em http://www.lawbydesign.co/en/ legal-design/>.

Programa de Gestão e Inovação - iNovaJusp, disponível em: <http://www.jfsp.jus.br/ documentos/administrativo/NUID/inovajusp/inovajuspcatalogada.pdf

ZAVAGLIA COELHO, Alexandre. As 7 tendências para o uso de inteligência artificial no direito. Thomson Reuters, Disponível em: <https://www.thomsonreuters.com.br/ content/dam/openweb/documents/pdf/Brazil/white-paper/As_7_Tend\%C3\%AAncias_ para_o_uso_da_Inteligencia_Artificial_EM_2018.pdf>.

ZAVAGLIA COELHO, Alexandre. A ciência de dados e a inteligência artificial no Direito. Dispnível em: https://www.conjur.com.br/2019-jan-01/zavaglia-ciencia-dados-inteligenciaartificial-direito. 